\title{
Effect of Pressure on the Relation between Glass Transition Temperature and Molecular Weight for Monodispersed Polystyrenes
}

\author{
Hirotoshi Toratani* and Kanichiro Takamizawa \\ Department of Applied Science, Faculty of Engineering, Kyushu University, \\ Hakozaki, Higashi-ku, Fukuoka 812 Japan
}

(Received September 22, 1993)

\begin{abstract}
The glass transition temperatures $T_{\mathrm{g}}$ of monodispersed polystyrenes possessing different number-averaged molecular weights $M_{n}$ were determined by high pressure DTA of up to $500 \mathrm{MPa}$. The measurements were performed using samples glassified at a cooling rate of $20 \mathrm{~K} / \mathrm{min}^{-1}$ under differing pressures. $T_{\mathrm{g}}$ was satisfactorily fitted against $P$ according to the Simon-type equation, which is usually applied to the relation between melting points and $P$. The linear relation of $T_{\mathrm{g}}$ against $M_{n}{ }^{-1}$ was found to hold even under high pressure. The slope of this relation increased from $0.98 \times 10^{5} \mathrm{~K}$ at atmospheric pressure to $1.19 \times 10^{5} \mathrm{~K}$ at $500 \mathrm{MPa}$. When the slope was analyzed by means of Bueche's model, the excess free volume of the chain ends decreased with increasing pressure and seemed to have an asymptotic value above $350 \mathrm{MPa}$, which was one-third of the value at atmospheric pressure.

KEY WORDS Glass Transition Temperature / Molecular Weight Dependence / Polystyrene / Free Volume / Pressure /
\end{abstract}

The linear relation of $T_{\mathrm{g}}$ against $M_{n}{ }^{-1}$ has been established for many linear polymers, ever since the fundamental work by Fox and Flory ${ }^{1}$ was published. The relation is represented by:

$$
T_{\mathrm{g}}=T_{\mathrm{g} \infty}-K / M_{n}
$$

where $T_{\mathrm{g} \infty}$ corresponds to the $T_{\mathrm{g}}$ of a polymer with infinite molecular weight. The slope $K$ represents the characteristics of the polymer chain. For monodispersed polystyrene, two studies using $\mathrm{DTA}^{2}$ and dilatometry ${ }^{3}$ have been reported. Although these studies were based on different properties of heat capacity and volume, and although the freezing-in rate of glassification differed greatly, the $K$ values which were obtained were nearly identical. The difference in $T_{\mathrm{g} \infty}$ could be reasonably interpreted in terms of the freezing-in rate, based on a linear correlation between $T_{\mathrm{g}}$ and the logarithm of the glassification rate. ${ }^{4,5}$ It has, moreover, been noted that an almost identical value of $K$ was also reported for the molecular weight fractions of thermally polymerized polystyrenes. ${ }^{1}$ It is concluded that $K$ can be used as a reliable parameter reflecting chain character.

Many studies on the pressure change of $T_{\mathrm{g}}$ have been carried out, partly because they would provide information which could shed light on the intrinsic nature of polymeric glass. However, it would seem that few studies on the pressure dependence of $K$ have been carried out. Using high pressure DTA, we have continued to study the relation of $T_{\mathrm{g}}$ vs. $M_{n}{ }^{-1}$ under high pressure DTA. ${ }^{5-8}$ Because DTA is sensitive and convenient, it enables experi-

* To whom correspondence should be addressed. Present Address: Tire Materials Development Department, Bridgestone Corporation, 3-1-1, Ogawahigashi-cho, Kodaira-shi, Tokyo 187, Japan. 
mental data on $T_{\mathrm{g}}$ under high pressure to be collected easily. In the present work, the glass transition temperatures of four anionic polystyrenes, whose molecular weights ranged from about $4 \times 10^{3}$ to $200 \times 10^{3}$, were determined under pressure of up to $500 \mathrm{MPa}$. Equation 1 was found to hold under pressures, and the pressure dependence of $K$ is presented. Obtained $K$ values could be used to interpret the physical nature of the polystyrene chain under high pressure.

$K$ has been analyzed by several theoretical treatments. The important ones being, a) an excess free volume treatment by Bueche, ${ }^{9}$ b) Somcynsky-Patterson's treatment based on the theorem of corresponding states, ${ }^{10}$ and c) a statistical thermodynamic theory by Gibbs and DiMarzio. ${ }^{11}$ Among them, Bueche's treatment is the most intuitive and seems to be applicable under high pressure without presenting any special difficulties. In this study, we determine the pressure dependence of the excess free volume of the chain ends by using the reported relations of $V_{\mathrm{g}} \Delta \alpha$ against $P$, where $V_{\mathrm{g}}$ is the volume at $T_{\mathrm{g}}$ and $\Delta \alpha$ is the difference in isobaric volume expansivity between the supercooled liquid and the glass.

\section{EXPERIMENTAL}

\section{Samples}

Four anionic polystyrenes purchased from Toso Corp. were used. According to the manufacturer's specifications, their $M_{n}$ s were $3900,6800,19400$, and $183 \times 10^{3}$ and their ratios of $M_{w} / M_{n}$ were 1.06, 1.04, 1.01, and 1.04, respectively. These samples were designated as PS04, PS07, PS20, and PS200 respectively. The samples were thoroughly degassed at $160^{\circ} \mathrm{C}$ for $2 \mathrm{~h}$ in order to eliminate volatile impurities. This treatment was needed to prevent the lowering of $T_{\mathbf{g}}$ by such impurities.

\section{DTA Apparatus}

The details of the high pressure DTA cell and the pressure system were described in a previous paper. ${ }^{12}$ A sample of approximately $6 \mathrm{mg}$ was punched out from a pressed sheet, placed in a platinum-alloy cup of the DTA sensor and covered with a thin lead lid. The gap between the cup and the lid was completely sealed with epoxy resin. An Alumel-Chromel thermocouple was spot-welded to the bottom of the cup. This may have caused a reduction in the heat capacity of the sample container used in the present experiment, when compared to the heat capacity of the container used in previous experiments. ${ }^{12}$ Lead was used as a reference material. The pressure-transmitting liquid was dimethylsilicone oil, whose viscosity was approximately $10 \mathrm{cS}$. When thermal noise became severe, within the low pressure range, an oil of high viscosity was used. Pressure was measured by a Manganin gauge, which was calibrated with a Heise precision gauge. Temperature calibration was determined by using the relation of the melting point of indium to pressure.

The $T_{\mathrm{g}}$ at atmospheric pressure was measured by a Rigaku DSC-8240B (a heat-flux type DSC with high sensitivity). Temperature calibration was determined by indium.

The data obtained by both instruments were stored in a personal computer (NEC8001MkII) and were used for data analysis.

\section{$T_{\mathrm{g}}$ Determination}

From the viewpoint that glass transition is a frozen-in process, it is reasonable to determine $T_{\mathrm{g}}$ through the change in volume or heat capacity during the cooling process. $T_{\mathrm{g}}$ is customarily defined as the middle point or the inflection point of the transition sigmoid on a cooling DTA/DSC curve. However, a cooling run under high pressure is often disturbed probably due to heat convection in the pressure transmitting liquid. On the other hand, a heating run usually provides a good DTA curve. Therefore the $T_{\mathrm{g}}$ measurement was carried out as follows:

The sample was glassified at a cooling rate of $20 \mathrm{~K} \mathrm{~min}^{-1}$, from $60 \mathrm{~K}$ above an estimated 
$T_{\mathrm{g}}$ at the particular pressure used, to $60 \mathrm{~K}$ below the $T_{\mathrm{g}}$. After a steady state was established, a heating run was started at a rate of $20 \mathrm{~K} \mathrm{~min}^{-1}$. Because the heating DTA curve usually shows slight superheating, the $T_{\mathrm{g}}$ was taken as the intersection temperature at which an extrapolated baseline of glass crosses over the initial base line and the sloping portion. The $T_{\mathrm{g}}$ point approximately conformed with

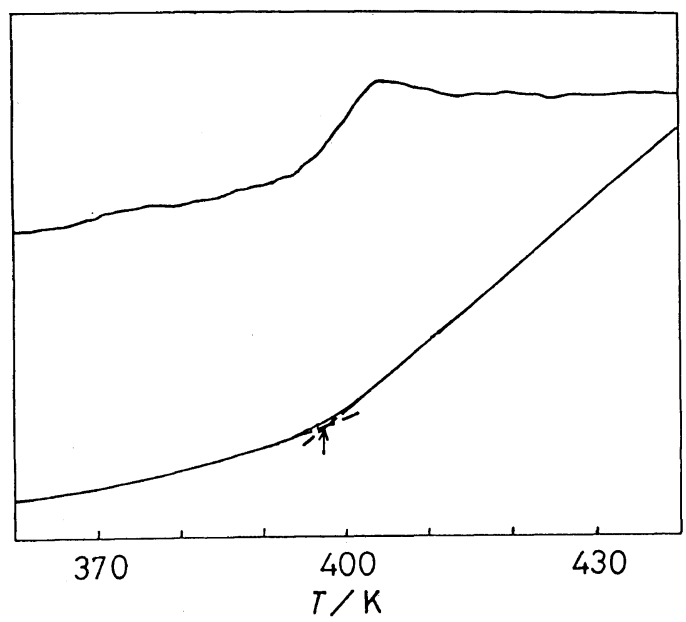

(a)

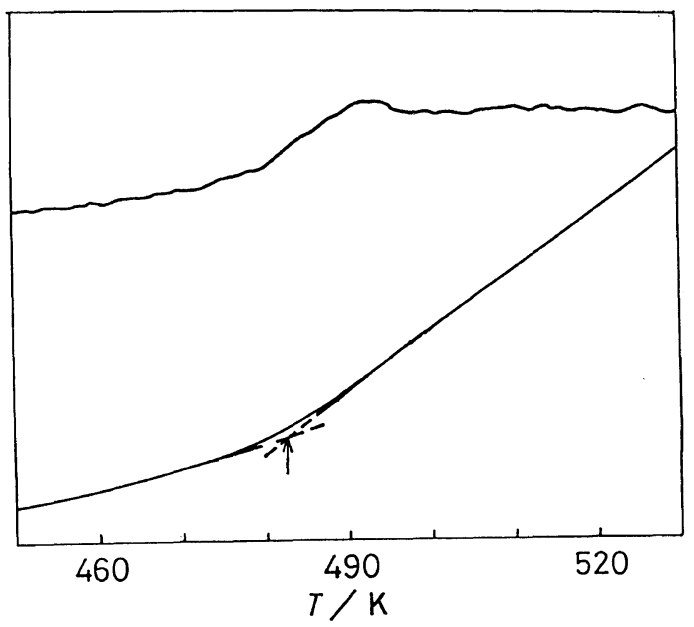

(b)

Figure 1. Heating DTA curve (top curve) and the integrated curve (bottom curve) for monodispersed polystyrene PS07: (a) at $100 \mathrm{MPa}$, and (b) at $500 \mathrm{MPa}$. The glassification rate is $20 \mathrm{~K} \mathrm{~min}^{-1}$ and the scanning rate is $20 \mathrm{~K} \mathrm{~min}^{-1}$. The arrow indicates $T_{\mathrm{g}}$ determined. the value determined by the cooling run.

Furthermore, in order to get the $T_{\mathrm{g}}$ obtained by DTA to conform to the one obtained by dilatometry, the following procedure was adopted:

An integrated curve was calculated from the DTA data for which the intersection temperature of the liquid and the glass portions was used as $T_{\mathrm{g}}$. The validity of this procedure has already been proven by Richardson and Savill. ${ }^{4}$ Because our DTA apparatus is a heat-flux type DSC,${ }^{13}$ this integration seems to be applicable.

In Figures 1(a) and 1(b), the heating DTA curve (top curve) and its integrated curve (bottom curve) at 100 and $500 \mathrm{MPa}$ are shown respectively. Figure 1 shows that the $T_{\mathrm{g}}$ point determined from the integrated curve approximately corresponds to that obtained from the DTA curve as described above. From a comparison between the DTA curves at 100 and $500 \mathrm{MPa}$, both of which were drawn on the same scale of the ordinate, it would seem that $\Delta C_{\mathrm{p}}$, the difference in heat capacity between liquid and glass decreases with increasing $P$. The decrease in the temperature difference at $T_{\mathrm{g}}$ arises from the increase in the thermal conductivity of the pressure-transmitting liquid at a high pressure. ${ }^{13}$ The data obtained at atmospheric pressure were treated similarly.

\section{RESULTS AND DISCUSSION}

Although many studies on the effect of pressure on $T_{\mathrm{g}}$ have been reported, the glassification conditions were ambiguous in most of these cases. The glassification condition is defined by the cooling rate at constant pressure, or by the pressurizing rate at a constant temperature. Control of the cooling rate is easier than that of the pressurizing rate. Only Oels and Rehage have reported $P V T$ experiments for monodispersed polystyrene in which isobaric cooling glassification was adopted. ${ }^{14}$ Moreover, in the present work, the $T_{\mathrm{g}}$ was determined on glassification by the same cooling rate at each of the pressures used. 
In Figure 2, $T_{\mathrm{g}}$ is plotted against $P$ for four different monodispersed polystyrenes. The dependence of $T_{\mathrm{g}}$ on $P$ is usually represented by polynomials of $P$ :

$$
T_{\mathrm{g}}=T_{\mathrm{g} 0}+A P+B P^{2}
$$

where $T_{\mathrm{go}}$ is $T_{\mathrm{g}}$ at atmospheric pressure, and where $A$ and $B$ are constants. As another example we could cite the Simon-type equation:

$$
T_{\mathrm{g}}=T_{\mathrm{g} 0}(1+P / a)^{1 / c}
$$

where $a$ and $c$ are constants. The Simon-type equation has been used empirically to represent the data of melting points at various pressures. Since both the equations are empirical, their appropriateness has to be judged from the fit of the data. In the present case, eq 3 provided a lower value of the sum of squares of residuals than eq 2 did. The method of a non-linear

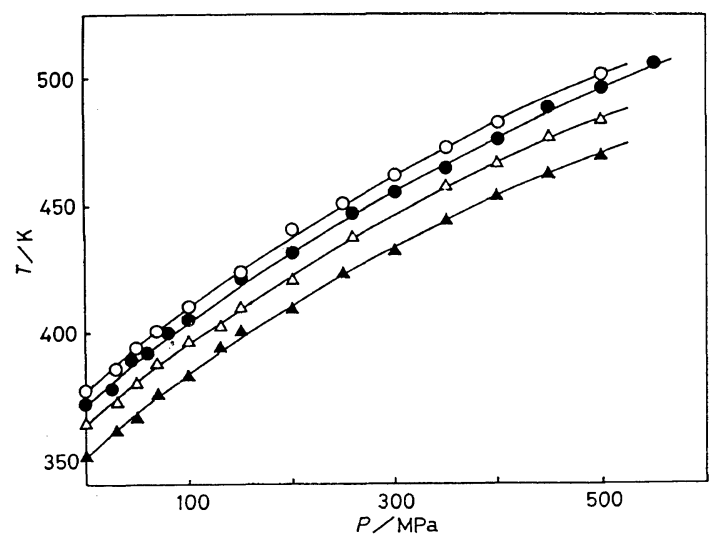

Figure 2. Pressure dependence of $T_{\mathrm{g}}$ for monodispersed polystyrenes glassified at a rate of $20 \mathrm{~K} \mathrm{~min}^{-1}$ at each of the pressures used. O, PS200;, PS20; $\triangle$, PS07; PS04. least-squares fitting of eq 3 was described in a previous paper. ${ }^{15}$

Although eq 3 is said to be empirical, it can be derived based on the following two assumptions:

a) one of the Ehrenfest equations on the pressure dependence of $T_{\mathrm{g}}$ holds:

$$
\mathrm{d} \ln T_{\mathrm{g}} / \mathrm{d} P=V_{\mathrm{g}} \Delta \alpha / \Delta C_{\mathrm{p}}
$$

b) the inverse of the right-hand side is approximated as a linear function of $P$ :

$$
\Delta C_{\mathrm{p}} /\left(V_{\mathrm{g}} \Delta \alpha\right)=c(P+a)
$$

While eq 4 is generally recognized, the second assumption seems to be valid for experimental $P V T$ data of polystyrene. Details of this derivation are described elsewhere. ${ }^{16}$

The solid curves in Figure 2 are drawn by using eq 3. The least-squares fitting was obtained with a fixed value of $T_{\mathrm{g} 0}$. The $T_{\mathrm{g} 0}$ value was calculated from the linear relation between $T_{\mathrm{g}}$ and the logarithm of the cooling rate at atmospheric pressure. Table I lists the $T_{\mathrm{g} 0}, a$, and $c$ for four different polystyrenes. The values of $a$ and $c$ are nearly independent of molecular weight. Table I also contains the pressure coefficients of $T_{\mathrm{g}}$ at atmospheric pressure and $400 \mathrm{MPa}$, which were calculated by using the constants. Moreover, these values also do not show any dependence on molecular weight. The value of the initial slope, $39 \mathrm{~K}$ per $100 \mathrm{Mpa}$, is larger than the average of the reported values, $31 \mathrm{~K}$ per $100 \mathrm{MPa} .{ }^{14,17-19}$ The higher value may be partly due to the use

\begin{tabular}{|c|c|c|c|c|c|}
\hline \multirow{2}{*}{ Sample } & \multirow{2}{*}{$T_{\mathrm{go}} / \mathrm{K}$} & \multirow{2}{*}{$a / \mathrm{MPa}$} & \multirow{2}{*}{$c$} & \multicolumn{2}{|c|}{$\mathrm{d} T_{\mathrm{g}} / \mathrm{d} P / \mathrm{K} / 100 \mathrm{MPa}$} \\
\hline & & & & $0.1 \mathrm{MPa}$ & $400 \mathrm{MPa}$ \\
\hline PS04 & 351.5 & $239.4 \pm 16.0$ & $3.85 \pm 0.17$ & 38.2 & 18.5 \\
\hline PS07 & 362.3 & $231.3 \pm 13.8$ & $3.91 \pm 0.15$ & 39.0 & 18.7 \\
\hline PS20 & 369.9 & $246.1 \pm 13.0$ & $3.78 \pm 0.13$ & 39.7 & 19.5 \\
\hline PS200 & 377.1 & $262.0 \pm 20.0$ & $3.76 \pm 0.19$ & 38.3 & 19.4 \\
\hline
\end{tabular}
of eq 3 . This high value may be useful when examining the validity of the Ehrenfest equa-

Table I. Constants of the Simon equation and the pressure coefficients of $T_{\mathrm{g}}$ for polystyrenes 
tions.

In Figure 3, $T_{\mathrm{g}}$ is plotted against $M_{n}{ }^{-1}$ at indicated pressures. The $T_{\mathrm{g}}$ were calculated using eq 3 and the constants in Table I. A linear relation of eq 1 satisfactorily holds at elevated pressures of up to $500 \mathrm{MPa}$. The pressure

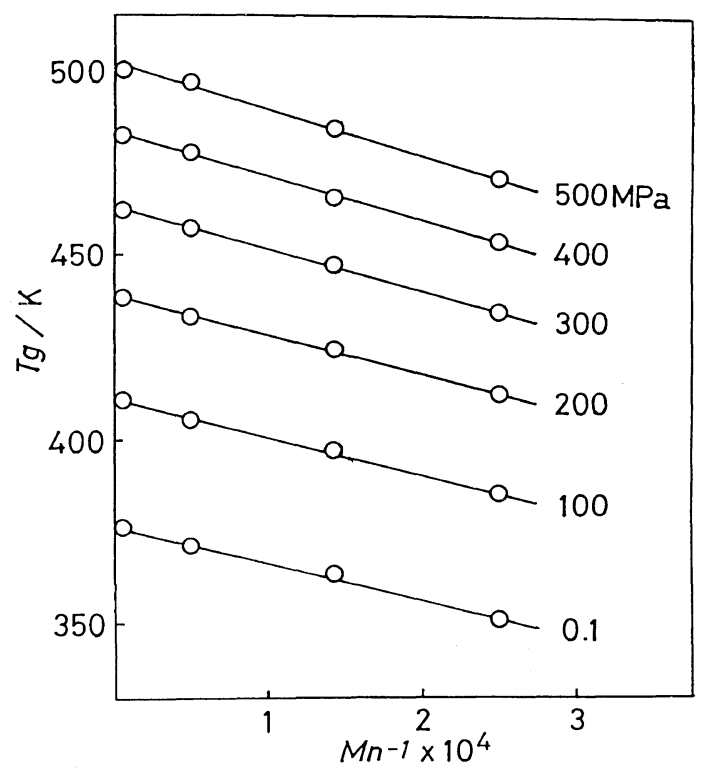

Figure 3. Plots of $T_{\mathrm{g}} v s{M_{n}}^{-1}$ at $100 \mathrm{MPa}$ intervals. The data were calculated using the Simon-type equation with the constants listed in Table I.

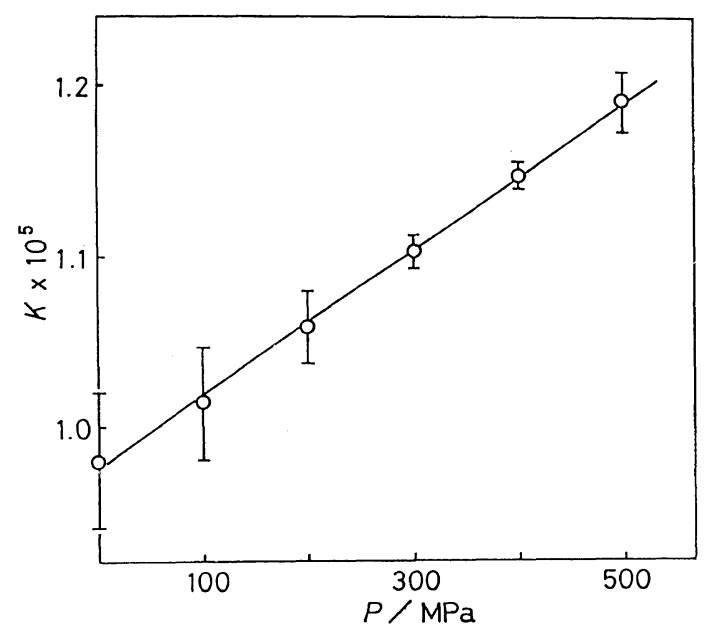

Figure 4. Pressure dependence of the slope $K$ indicating the linear relationship between $T_{\mathrm{g}}$ and $M_{n}{ }^{-1}$; the bars indicate the probable error. change of the slope $K$ is shown in Figure 4 (the bar indicates the probable error). $K$ increases almost linearly from $0.98 \times 10^{5} \mathrm{~K}$ at atmospheric pressure to $1.19 \times 10^{5} \mathrm{~K}$ at $500 \mathrm{MPa}$. The atmospheric value is in good agreement with $0.84 \times 10^{5} \mathrm{~K}$ for glass vitrified at 20 $\mathrm{K} \mathrm{min}^{-1}$, and with $1.03 \times 10^{5} \mathrm{~K}$ for glass vitrified at $2.5 \mathrm{~K}$ per day. ${ }^{2,3}$ The linear increase of $K$ with $P$ seems to be somewhat puzzling, although, its increase is relatively small.

$K$ can be easily analyzed in terms of the free volume concept. ${ }^{1,9}$ Assuming that the free volume at $T_{\mathrm{g}}$ is constant for samples with different molecular weights, $K$ is given by:

$$
K=2 \theta N_{\mathrm{A}} /\left(V_{\mathrm{g}} \Delta \alpha\right)
$$

where $2 \theta$ is the excess free volume of the chain ends per molecule and $N_{\mathrm{A}}$ the Avogadro number. $V_{\mathrm{g}}$ is used as an easily definable quantity. The product $V_{\mathrm{g}} \Delta \alpha$ under high pressure was reported by Oels and Rehage ${ }^{14}$ and also by Quach and Simha. ${ }^{17}$ In the study by Quach and Simha, the pressure range was limited to $200 \mathrm{MPa}$ and the data were obtained

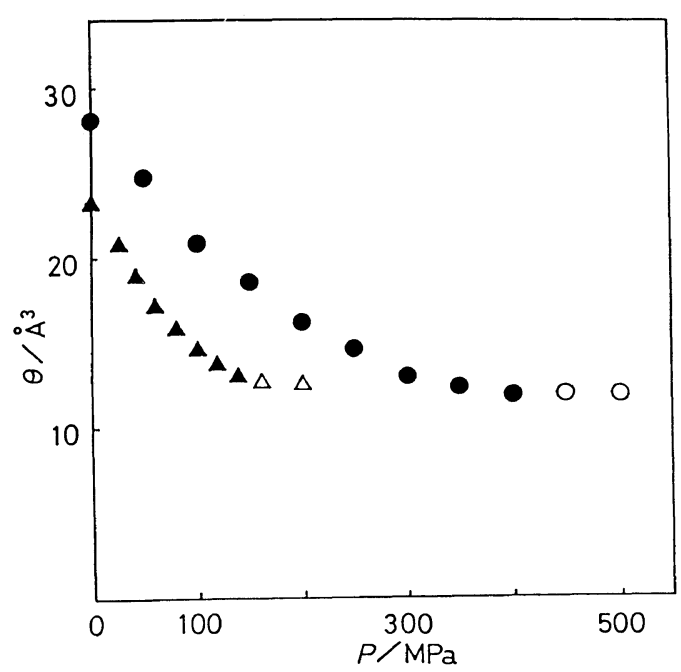

Figure 5. Pressure change in the excess free volume of the chain ends. $\theta$ was analyzed by Bueche's eq 6 . Symbols indicate the difference in $V_{\mathrm{g}} \Delta \alpha$ used. Open symbols refer to the use of extrapolated values. $\bigcirc$ and $\bigcirc$ are based on the data of Oels and Rehage, ${ }^{14}$ while $\Delta$ and $\Delta$ are based on the data of Quach and Simha. ${ }^{17}$ 
by isothermal compression. On the other hand, Oels and Rehage measured the volume change with an isobaric cooling process of up to $400 \mathrm{MPa}$. However, the data for $V_{\mathrm{g}}(P)$ by the two groups are nearly identical. As described in the previous paper, ${ }^{5}$ the $T_{\mathrm{g}}$ of Oels and Rehage is lower than that of our data, even if the effect of the freezing-in rate is taken into consideration. However, the lowering of $T_{\mathrm{g}}$ does not seem to have any effect on $V_{\mathrm{g}}$.

The pressure change of $\theta$, the excess free volume per chain end is shown in Figure 5, in which circles are the values calculated by using the data of Oels and Rehage and triangles indicate the values calculated using the data of Quach and Simha. Open symbols are used to indicate the $\theta$ values calculated by relations extrapolated to higher pressures. The products $V_{\mathrm{g}} \Delta \alpha$ calculated using the data of Oels and Rehage, and of Quach and Simha decrease rapidly with increasing $P$. However, $K$ is less sensitive on $P$ as shown in Figure 4. Therefore, the change in $\theta$ is remarkably dependent on the product. This shows the importance. of obtaining reliable $P V T$ data concerning the glass transition. The data of Quach and Simha reveal a larger pressure dependence than the data of Oels and Rehage. If we place the reliability of $V_{\mathrm{g}} \Delta \alpha$ on the data of Oels and Rehage, then $\theta$ approaches an asymptotic value at pressures greater than $350 \mathrm{MPa}$, which is about one-third of the value at atmospheric pressure.

According to an intuitive interpretation of $\theta$, Figure 5 implies that the excess free volume of the chain ends is more compressible than the free volume of the repeating unit. This implication may agree with an intuitive image of the chain ends. If we adopt the data of Quach and Simha, the end becomes far softer. Pezzin et $a .^{20}$ suggested that in order to explain the behavior of $\theta$ for many kinds of polymers, it would seem reasonable to take into account the molecular flexibility rather than the difference in the chain ends. This may be a reasonable suggestion, since the meaning of the excess is relative to the entity of the chains. In order to obtain a better understanding of this subject, we must determine the relationship between $\theta$ and $P$ for other polymers.

The treatment by Somcynsky and Patterson ${ }^{10}$ led to the following equation:

$$
K=T_{\mathrm{g} 0} M_{0}(\beta / \alpha)
$$

where $M_{0}$ is the molecular weight of the repeating unit and the ratio of $\beta / \alpha$ is a quantity which decreases with increasing chain flexibility. The ratio of $\beta / \alpha$ decreases with increasing pressure. This may contradict the interpretation, since it seems unreasonable that the flexibility increases with pressure. This model is said to be too simple to be applied under high pressure.

When the theory of Gibbs and DiMarzio ${ }^{11}$ is applied, we are presented with the problem that the effect of pressure on the relation between the characteristic temperature $T_{2}$ and the observed $T_{\mathrm{g}}$ is unknown. According to our previous results, ${ }^{5}$ the effect of the freezing-in rate on $T_{\mathrm{g}}$ depends on $P$. This may be reflected by the relationship between $T_{2}$ and $T_{\mathrm{g}}$ at different pressures.

\section{REFERENCES}

1. T. G. Fox and P. J. Flory, J. Appl. Phys., 21, 581 (1950); J. Polym. Sci., 14, 315 (1954).

2. L. A. Wall, Roestamjah, and M. H. Aldridge, J. Res. Natl. Bur. Std., 78A, 447 (1974).

3. M. J. Richardson and N. G. Savill, Polymer, 18, 3 (1977).

4. M. J. Richardson and N. G. Savill, Polymer, 16, 753 (1975).

5. K. Takamizawa and H. Toratani, Thermochim. Acta, 163, 325 (1990).

6. K. Takamizawa and F. E. Karasz, presented at the State-of-the-Art of Thermal Analysis, NBS, May 1979. Experimental data were cited in ref 7.

7. K. Takamizawa, Y. Nagao, and Y. Urabe, Kyushu Daigaku Sogorikogakukenkyuka Hokoku (Eng. Sci. Reports, Kyushu Univ.), 6, 163 (1985).

8. K. Takamizawa and H. Toratani, Polymer, 33, 1333 (1992).

9. F. Bueche, "Physical Properties of Polymers," Interscience, New York, N.Y., 1962.

10. T. Somcynsky and D. Patterson, J. Polym. Sci., 62, 
151 (1962).

11. J. H. Gibbs and E. A. DiMarzio, J. Chem. Phys., 28, 373 (1958).

12. K. Takamizawa, Y. Nagao, D. Irii, and Y. Urabe, Thremochim. Acta, 88, 205 (1985).

13. K. Takamizawa, Thermochim. Acta, 25, 1 (1978).

14. H.-J. Oels and G. Rehage, Macromolecules, 10, 1036 (1977).

15. K. Takamizawa, A. Ohno, and Y. Urabe, Polym. J., 7, 342 (1975).
16. K. Takamizawa, H. Toratani, and F. E. Karasz, Thermochim. Acta, submitted.

17. A. Quach and R. Simha, J. Appl. Phys., 42, 4592 (1971)

18. S. Ichihara, A. Komatsu, and T. Hata, Polym. J., 2, 650 (1971).

19. J. R. Stevens, R. W. Coakley, K. W. Chau, and J. L. Hunt, J. Chem. Phys., 84, 1006 (1986).

20. G. Pezzin, F. Zilio-Grandi, and P. Sanmartin, Eur. Polym. J., 6, 1053 (1970). 